Original Article

\title{
Effects of isometric contraction of the affected-side upper limb in the supine position on the opposite side muscle activity of the body trunk muscles of normal adults
}

\author{
Yoonhee Chol, MS, $\mathrm{PT}^{1)}$, Sangyong Lee, $\mathrm{PhD}, \mathrm{PT}^{2)^{*}}$ \\ 1) Department of Physical Therapy, Graduate School, Deajeon University, Republic of Korea \\ 2) Department of Physical Therapy, U1 University: 12 Youngdong-eup, Youngdong-gun, \\ Chungbuk 370-701, Republic of Korea
}

\begin{abstract}
Purpose] This study examines the effect of isometric contraction of the upper limb on the affected side in the supine position on the activity of body trunk muscles on the opposite side of normal adults. [Participants and Methods] The research participants included 10 normal adults. A handheld dynamometer device was used to measure the isometric contraction of the upper limb. The muscle activity of the body trunk was analyzed by electromyography. [Results] The muscle activities of rectus abdominis, internal oblique abdominis, erector spinae muscle, and multifidus muscle of the body trunk on the opposite side were significantly larger in the case of the adduction and flexion of the shoulder joint on the affected side than in the case of abduction and extension. [Conclusion] The isometric contraction induced by the adduction and flexion of the shoulder joint on the affected side altered the muscle activity of the body trunk on the opposite side.

Key words: Electromyography, Isometric contraction, Trunk muscles
\end{abstract}

(This article was submitted Jun. 12, 2018, and was accepted Aug. 2, 2018)

\section{INTRODUCTION}

Stroke is a cerebrovascular disease in which decreased consciousness and body paralysis suddenly occur because of a circulatory disturbance. The segment that stroke patients occupy in the rehabilitation is increasing ${ }^{1)}$. As an exercise treatment method from the neurological aspect, diverse types of direct therapeutic approaches to the functional recovery of the affected side have been implemented so far. A recent study reported that an indirect therapeutic approach that aims at the recovery of the affected side by using the body part on the unaffected side was effective ${ }^{2)}$. The study of Hellebrandt et al. ${ }^{3)}$, which is related to indirect therapy, reported that the maximum exercise of the body trunk and limbs on the affected side causes muscle tension of the body part on the opposite side that did not exercise. They also reported that the flexion pattern of the upper limb could indirectly activate the body trunk muscles as it causes the contraction of abdominal muscles. Stroke patients experience selective posture abnormality, difficulties in motion control, and inappropriate muscle tension. They also have an abnormal symmetry of the body trunk and stability of the proximal part because of paralysis of one side of the body. Abnormal posture causes asymmetric alignment, which affects the stability of the body trunk. As a result, posture maintenance and walking become difficult ${ }^{4}$. Although several previous studies were conducted on the functional disability of the upper and lower limb muscles among hemiplegic patients due to stroke ${ }^{5,6)}$, reports related to body trunk muscles are rare. Moreover, domestic studies in Korea that examine the effect of isometric muscle contraction of the affected-side upper limb on the muscle activity of the opposite-side body trunk are scarce. This study investigates the change in muscle activity of the body trunk on the

*Corresponding author. Sangyong Lee (E-mail: 1sy8275@hanmail.net)

(C2018 The Society of Physical Therapy Science. Published by IPEC Inc.

(c) (1) $($ This is an open-access article distributed under the terms of the Creative Commons Attribution Non-Commercial No Derivatives cC. 
Table 1. Comparison of the muscle activities of body trunk muscles on the opposite side according to the isometric contraction of the affected-side upper limb (unit: \%)

\begin{tabular}{lcccrl}
\hline & \multicolumn{2}{c}{ Isometric contraction of the affected-side upper limb } & \\
\cline { 2 - 4 } & Abduction & Adduction & Flexion & Extension & \\
\hline RA $^{* *}$ & $10.0 \pm 6.9$ & $17.4 \pm 8.4$ & $15.5 \pm 6.8$ & $7.9 \pm 5.6$ & Abduction, Extension < Adduction, Flexion \\
IOA $^{*}$ & $16.1 \pm 6.1$ & $20.1 \pm 7.6$ & $18.3 \pm 8.5$ & $11.1 \pm 6.5$ & Abduction, Extension < Adduction, Flexion \\
ES $^{* *}$ & $11.9 \pm 4.1$ & $21.8 \pm 9.7$ & $19.9 \pm 8.7$ & $8.7 \pm 5.1$ & Abduction, Extension < Adduction, Flexion \\
Mf $^{*}$ & $14.8 \pm 8.2$ & $23.6 \pm 8.5$ & $18.2 \pm 5.2$ & $11.3 \pm 9.0$ & Abduction, Extension < Adduction, Flexion \\
\hline
\end{tabular}

Date are presented as mean $\pm \mathrm{SD}$, RA: rectus abdominis; IOA: internal oblique abdominis; ES: Erector spinae muscle; Mf: multifidus muscle, ${ }^{*} \mathrm{p}<0.05, * * \mathrm{p}<0.01$, *repeated one-way ANOVA.

opposite side according to the isometric contraction of the affected-side upper limb of normal people.

\section{PARTICIPANTS AND METHODS}

A total of 10 healthy adults (four males and six females) enrolled in University U located in Chungcheongbuk-do Province, South Korea, were selected as the research participants of this study. Average age, average weight, and average height of the research participants were $21.3 \pm 1.5$ years, $56.5 \pm 10.6 \mathrm{~kg}$, and $168.4 \pm 8.2 \mathrm{~cm}$, respectively. The participants with surgical or neurological damages were excluded from the sample. Ethical approval for the study was granted by the U1 University institutional review board (U1IRB2018-12). All participants read and signed the consent forms in accordance with the ethical standards of the Declaration of Helsinki.

A handheld dynamometer device (Commander ${ }^{\text {TM }}$, JTECH medical, Salt Lake City, UT, USA) was used to measure the isometric contraction of the upper limb The MP150 (BIOPAC System Inc. Santa Barbara, CA, USA) surface electromyogram (EMG) system was used to measure the change in muscle activity. The surface electrode was attached to the rectus abdominis (RA), internal oblique abdominis (IOA), erector spinae muscle (ES), and multifidus muscle (Mf). The average value of the EMG signal of each participant was expressed as the percentage of the maximum voluntary isometric contraction.

To perform the initial posture, the research participants maintained the anatomic posture in the supine position as they put their feet shoulder width apart and took a neutral position of their toes. To conduct a homogeneous experiment method for all the participants, we measured the muscle activity of the RA, IOA, ES, and Mf of the non-dominant-side body trunk during the isometric contraction of abduction (Abd), adduction (Add), flexion (flex), and extension (ext) of the shoulder joint of the dominant-side upper limb. The order of the four types of shoulder joint exercises applied to the participants was randomly determined. We used a handheld dynamometer device to identically provide the participants with proper resistance. Resistance was given so that $50 \%$ of the maximum contraction was maintained. Resistance was applied to the outside, inside, front, and rear of the wrinkle and the distal part of the lower arm during the isometric contraction of the shoulder joint, which includes Abd, Add, flex, and ext. To maintain constant resistance steadily for $5 \mathrm{~s}$, visual feedback was provided to the patients and the evaluator using a handheld dynamometer device. A 1 min resting time was given between each exercise. Contraction for $5 \mathrm{~s}$ was repeated for three times, and a 5 min resting time was given between each exercise. The average muscle activity was computed to be used in the final analysis.

For the statistical treatment in this study, a repeated one-way ANOVA was conducted to investigate the muscle activity of the opposite-side lower limbs according to the isometric contraction of the affected-side upper limb. The Bonferroni correction method was used for the post-hoc test. The Excel 365 program (Microsoft office Inc., Korea) was used for statistical treatment. A statistically significant level was set to $\alpha=0.05$

\section{RESULTS}

All of the Abd, Add, flex, and ext of the shoulder joint of the affected-side upper limb produced a significant difference in the muscle activity of RA, IOA, ES, and Mf of the body trunk on the opposite side $(\mathrm{p}<0.05)$. In the post-hoc test, the Add and flex of the shoulder joint of the affected-side upper limb showed a significantly higher muscle activity of the RA, IOA, ES, and Mf of the body trunk on the opposite side than the Abd and ext $(\mathrm{p}<0.05)$ (Table 1).

\section{DISCUSSION}

Tarnanen et al. ${ }^{7)}$ applied isometric contraction of the upper limb to both sides or one side in a standing position and examined the muscle activity of the body trunk on the same side and the opposite side. They reported that the muscle activity of Mf on the same side and the opposite side increased the most in the case of the horizontal abduction of the shoulder joint. Crommert et al. ${ }^{8)}$ found that supporting one's weight in a standing position while horizontally stretching the arms at shoulder 
joint height increased the muscle activity of ES more than in the case without stretching the arms. They also reported a significant effect on the muscle activity of the spinal extensor according to the location of the resistance loaded on the limbs. In this study, the isometric contraction of adduction and flexion of the upper limb induced a stronger muscle activity of the RA, IOA, ES, and Mf than abduction and extension. Myers et al. ${ }^{9)}$ argued that the entire human body reacts when one body part moves and that connective tissue is the only tissue that is capable of functional intervention. Their study addressed the interaction and the overall function of connective tissue, fascia, and other body systems using a line expression, such as front, rear, side, and oblique lines. Page et al. ${ }^{10)}$ categorized muscles where tension easily occurs and argued that the tonic muscle system is easily activated with adduction and flexion motions. The anterior deltoid and coracobrachialis muscles act in the flexion motion and muscle pectoralis major, latissimus dorsi muscle, and posterior deltoid act in the adduction motion. Here, the abdominal fascia chain acts as the IOA, external oblique abdominal muscle, muscle transversus abdominis, muscle pectoralis major, and muscle serratus anterior form a diagonal line. They also reported that the thoracolumbar fascia chain acts through the connection of the IOA, external oblique abdominal muscle, muscle transversus abdominis, latissimus dorsi muscle, and gluteus maximus muscle. Myers et al. ${ }^{9)}$ argued that the muscle contraction of the affected-side limbs transfers force to other muscles, tendon, fascia, ligament, articular capsule, and bone beyond the insertion and origin range of the contracting muscles. As a result, the muscle activity of the opposite-side body trunk that is far away is also affected through the body muscle system during the muscle contraction of the upper and lower limbs. Our study result, which demonstrated the effect of flexion and adduction motions in the case of the isometric contraction of the affected-side upper limb on the muscle activity of the RA, IOA, ES, and Mf of the opposite-side body trunk, is consistent with that of Page et al ${ }^{10)}$.

Our study restricted the number of participants, and the results cannot be generalized to every participant. Further studies are required in the future to investigate the muscle activity of the body trunk on the opposite side according to the movement of limbs using hemiplegic patients as a sample.

\section{Conflict of interest}

None.

\section{REFERENCES}

1) Seo JH, Ko MH, Kim YH: The effects of shoes modification on energy consumption in hemiplegic gait. Ann Rehabil Med, 1999, 23 : 17-23.

2) Kim JM, Lee CH: Neurological physical therapy. Jungdam, Seoul, 2001, pp 197-201.

3) Hellebrandt FA, Parrish AM, Houtz SJ: The influence of unilateral exercise on the contralateral limb. Arch Phys Med Rehabil, 1947, 28: 76-85. [Medline]

4) Shin JH, Oh DW, Kim JS: Effect of Nordic walking training on walking function of patients with stroke. J of Special Education \& Rehabilitation Scicence, 2010, 49: 181-194.

5) Adams RW, Gandevia SC, Skuse NF: The distribution of muscle weakness in upper motoneuron lesions affecting the lower limb. Brain, 1990, 113: 1459-1476. [Medline] [CrossRef]

6) Colebatch JG, Gandevia SC: The distribution of muscular weakness in upper motor neuron lesions affecting the arm. Brain, 1989, 112: 749-763. [Medline] [CrossRef]

7) Tarnanen SP, Ylinen JJ, Siekkinen KM, et al.: Effect of isometric upper-extremity exercises on the activation of core stabilizing muscles. Arch Phys Med Rehabil, 2008, 89: 513-521. [Medline] [CrossRef]

8) Crommert ME, Ekblom MM, Thorstensson A: Activation of transversus abdominis varies with postural demand in standing. Gait Posture, 2011, 33: 473-477. [Medline] [CrossRef]

9) Myers TW, Juhan D, Chaitow L: Anatomy train: myofascial meridians for manual and movement therapists, 1st ed. UK: Churchill1 Livingstone, Elservier Science, 2001.

10) Page P, Frank C, Lardner R: Assessment and treatment of muscle imbalance; The Janda approach, Human kinetics, 2010. 